\title{
Gestión del conocimiento en las
}

\section{Instituciones de Educación Superior}

\section{para la apropiación social del}

\section{conocimiento}

\author{
Gleidy-Alexandra Urrego-Estrada ${ }^{1}$ \\ Jahir-Alexander Gutiérrez-Ossa² \\ Diego-Armando Jurado-Zambrano ${ }^{3}$
}

Fecha de recepción: 15 de febrero de 2021

Fecha de aprobación: 15 de marzo de 2021

\section{Resumen}

El propósito del artículo es reflexionar acerca de la gestión del conocimiento en las Instituciones de Educación Superior en Colombia IES para la apropiación social del conocimiento, a través de las actividades de investigación para la transferencia de información y conocimiento en un área del saber. La ruta metodológica es de tipo cualitativa y está orientada en la reflexión analítica de asuntos y bases de la gestión del conocimiento en las IES para la apropiación social del conocimiento, a partir de establecer horizontes reflexivos que permitan comprenderla. Se desarrolló en dos momentos: En el primero, se realizarán discusiones conceptuales sobre categorías

\footnotetext{
M. Sc. Escuela Superior de Administración Pública - ESAP (Medellín-Antioquia, Colombia). gleidy.urrego@esap.edu.co. ORCID: 0000-0002-7040-0048

2 Ph. D. Escuela Superior de Administración Pública - ESAP (Medellín-Antioquia, Colombia). jahir.gutierrez@esap.edu.co. ORCID: 0000-0001-8564-0397

3 M. Sc. Escuela Superior de Administración Pública - ESAP (Medellín-Antioquia, Colombia). diego.jurado@esap.edu.co. ORCID: 0000-0001-6666-6444
} 
como: región del conocimiento y apropiación social del conocimiento. En el segundo momento se realizó el procesamiento y análisis de la información. Los resultados se centran hacia un nuevo enfoque donde la prioridad sea la transferencia como un proceso de intercambio y apropiación social del conocimiento para la innovación y transformación social. Como principal conclusión considera que la gestión del conocimiento implica para las IES el reconocerse como entidades que generan, impactan, transfieren y promueven el conocimiento como un proceso de intercambio, y no se limitan a su adquisición como sucede en la actualidad.

Palabras clave: capital humano; difusión de conocimientos; educación superior; gestión del conocimiento; transferencia de conocimientos.

\section{Knowledge Management in Higher Education Institutions for the Social Appropriation of Knowledge}

\section{Abstract}

The purpose of this article is to reflect on the management of knowledge in Higher Education Institutions in Colombia IES for the social appropriation of knowledge, through research activities for the transfer of information and knowledge in an area of knowledge. The methodological route is of a qualitative type and is oriented on the analytical reflection of issues and bases of knowledge management in HEls for the social appropriation of knowledge, based on establishing reflective horizons that allow understanding it. It was developed in two moments: In the first, conceptual discussions will be held on categories such as: region of knowledge and social appropriation of knowledge. In the second moment, the information processing and analysis was carried out. The results are focused on a new approach where the priority is transfer as a process of exchange and social appropriation of knowledge for innovation and social transformation. As the main conclusion, it considers that knowledge management implies for HEls to recognize themselves as entities that generate, impact, transfer and promote knowledge as a process of exchange, and are not limited to its acquisition as is currently the case.

Keywords: dissemination of knowledge; higher education; human resources; knowledge management; know-how transfer. 
Para citar este artículo:

Urrego-Estrada, G.-A., Gutiérrez-Ossa, J.-A., Jurado-Zambrano, D.-A. (2021). Gestión del conocimiento en las Instituciones de Educación Superior para la apropiación social del conocimiento. Pensamiento y Acción, 31, 27-51.

Esta obra está bajo licencia internacional Creative Commons Reconocimiento 4.0

(c) 


\section{Introducción}

El Instituto Colombiano para la Ciencia y la Investigación como alguna vez fue concebido Colciencias, ahora Minciencias es el organismo rector del Sistema Nacional de Ciencia, Tecnología e Innovación -SNCTI, el cual consolida la articulación entre el sector gubernamental, empresarial, académico y la sociedad civil mediante la generación, transferencia y apropiación del conocimiento. En este sentido, el Gobierno Nacional sancionó la Ley 1951 de 2019 la cual hizo posible la transformación de Colciencias al Ministerio de Ciencia, Tecnología e Innovación CTel -, con el fin de impulsar el Desarrollo y la competitividad en el país. E Ministerio de CTel Minciencias busca el fortalecimiento del Sistema Nacional de Ciencia, Tecnología e Innovación a través de la articulación entre universidades, el sector privado y el Estado, a la vez que promueve un buen relacionamiento con el Sistema Nacional de Competitividad e Innovación (SNCl).

En tal sentido, la gestión del conocimiento debe considerar los lineamientos de la Estrategia Nacional de Apropiación Social de la Ciencia (2009), la Política Nacional de Apropiación Social del Conocimiento de (2020) y el documento borrador CONPES de la Política de CTel (2021-2030). Por tanto, la creación de Ministerio le apuesta a responder a los retos del país en los siguientes cinco ejes: (i) Articular la relación Universidad - Empresa - Estado - Sociedad; (ii) Impulsar la generación de conocimiento, la transferencia y la innovación para el sector productivo y social; (iii) Promover la creación de empresas basadas en el conocimiento (Spin Off); (iv) Potenciar las capacidades de CTel regionales y sectoriales; y (v) Generar una cultura que valore, use y apropie el conocimiento y la innovación (Minciencias, 2019). De otro lado, criterios y fundamentos de mercado, tales como la competitividad, eficacia, eficiencia y la capacidad financiera, han obligado a las Instituciones de Educación Superior IES a repensar sus estructuras operativas y ampliar su campo de acción para atraer recursos y trascender el hecho de únicamente entregar mano de obra calificada al mercado. No obstante, son pocas las que se destacan por la gestión del conocimiento.

Es común, que las IES pertenecientes a los países desarrollados estén a la vanguardia a la hora de materializar resultados concretos en cuanto a gestión del 
conocimiento, caso contrario, a lo que sucede en los países emergentes o en vía de desarrollo. La estructura académica, docente e investigativa, propias de la autonomía de las IES han pasado a ser marco de referencia del mercado. El proceso de entrada de los indicadores, parámetros y variables de este último las ha puesto en escala de nivel gerencial y de gestión. No obstante, pese a la bondad de su composición ha sido poco observable el papel cumplido por las IES en materia de transferencia de conocimiento, de paso, la formación profesional no contempla el desarrollo de competencias para la gestión del conocimiento y menos para que los futuros profesionales se conviertan en actores activos en el proceso de apropiación del conocimiento.

El mercado ha permitido la aparición de ofertas académicas provenientes de la iniciativa privada, hecho que no discrepa las bases públicas sobre las que ha sido desarrollado el conocimiento y la investigación. En un principio, las IES de carácter público surtieron la demanda académica y profesional de distintos sectores, para luego, entrar de lleno en competencia con el sector privado, interesado en formar su propio profesional y de paso iniciar proyectos de orden investigativo en aras de transferirlos a las empresas. Al ubicar el centro de la tesis en el marco de referencia de las IES de carácter público, se pretende, ilustrar el contenido y margen de acción que puede tener la gestión del conocimiento en este tipo de instituciones, sobre la base del avance en educación y soporte en investigación. La discusión no estriba en la denominación institucional sino en la capacidad que tiene esta para internalizar los esfuerzos que se hacen en dichos terrenos y externalizar de manera positiva los frutos obtenidos.

La puja por recursos obliga a las IES a tener respuestas concretas con respecto a cada actividad que desarrollan. No bastan los recursos financieros fruto de la cobertura e ingreso de los estudiantes a las diferentes carreras, es imperante, el comprender la docencia e investigación como los puntos de partida para la gestión del conocimiento, lo cual lleva a reflexionar sobre el papel de la IES, el cual no termina con la entrega de ciudadanos competentes al mercado, sino que trasciende hacia generar los medios para que éstos se conviertan en actores de la transformación desde la investigación, la transferencia y la apropiación de 
conocimiento que conlleve al planteamiento de soluciones innovadoras a las problemáticas sociales.

En este sentido, el presente escrito aborda en un primer momento el marco teórico y la metodología la cual debe abordarse al momento de referirse a la gestión del conocimiento en las IES en Colombia, como una plataforma necesaria para propiciar la apropiación social del conocimiento y a partir de ésta innovaciones públicas. En un segundo momento, las discusiones, resultados y conclusiones se encaminan a reflexionar en torno a la gestión del conocimiento a partir de direccionar las actividades de investigación en cuanto a la capacidad de transferencia de información y resultados de investigación gestados en un área del saber de las IES hacia actores productivos, gubernamentales y de la sociedad civil.

\section{Metodología}

La ruta metodológica es de tipo cualitativa y está orientada en la reflexión analítica de asuntos y bases de la gestión del conocimiento en las IES para la apropiación social del conocimiento, a partir de establecer horizontes reflexivos que permitan comprenderla. Se desarrolló en dos momentos: En el primero, se realizarán discusiones conceptuales sobre categorías como: región del conocimiento y apropiación social del conocimiento. Para ello, el procedimiento a seguir fue búsqueda y revisión documental y bibliográfica en bases de datos especializadas como: ProQuest, EBSCO, Scopus, Alfaomega CLOUD, libros académicos. Para esta revisión se empleó fichas de revisión bibliográfica. En el segundo momento se realizó el procesamiento y análisis de la información mediante una discusión grupal en torno a la información complida y procesada, a fin de establecer una ruta de discusión sobre el papel que deben cumplir la investigación en las IES.

\section{Resultados}

La generación de conocimiento se ha configurado como una de las fuentes de ventaja comparativa entre países (Hidalgo \& Albors, 2008), al punto que en la economía mundial, es una realidad que el equilibrio entre el conocimiento y los recursos tangibles se ha desplazado hasta ahora hacia los primeros y que el 
conocimiento se ha convertido quizás en el factor más importante que determina el nivel de vida (Banco Mundial, 1998). Nonaka (2007) afirma que en una economía donde la única certeza es la incertidumbre, la única fuente segura de ventaja competitiva duradera es el conocimiento.

Dada la importancia del conocimiento para la organizaciones, hasta el punto de considerarlo como el recurso más importante de la organización moderna y por tanto fuente de su singularidad (Raj Adhikari, 2010) se ha visto la necesidad de gestionar de manera estructurada el mismo, esto es planificando sus implicaciones e impactos que se espera para la organización y sus grupos de interés. En este sentido, se empezó a hablar de gestión del conocimiento, la cual, según Laal (2011) la define como un proceso en el cual el conocimiento necesario para que una organización tenga éxito es creado, capturado, compartido y apalancado.

La gestión del conocimiento da cuenta de las estrategias y procesos de adquisición, conversión, aplicación y protección del conocimiento (Lin \& Lee, 2006) para mejorar la competitividad de una empresa (Lin \& Lee, 2006; Von Krogh, 1998) y para lograr los objetivos de la organización (Landoli \& Zollo, 2006; Chong et al., 2011). En este sentido, la gestión del conocimiento se convierte en el nuevo objetivo que debe ser perseguido por las IES, en la medida que se comprenda la posibilidad de que éstas, pasen de la formación y la tutela profesional a servir de cuna y plataforma para la generación y transferencia de conocimiento, respectivamente.

La gestión del conocimiento adquiere importancia en las IES, entre otros aspectos, porque contribuye a mejorar la gestión interna, promueve la innovación mediante la transferencia de conocimiento, permitiendo alcanzar un nivel superior de calidad que genera mayor valor organizacional (Escorcia \& Barros, 2020). Dentro de los estudios actuales Escorcia \& Barros (2020) señalan que la gestión del conocimiento es una "herramienta estratégica para las IES, dentro de su objetivo de generar ventajas competitivas sostenibles en sus funciones de docencia, investigación y extensión"(p.92). En el mismo sentido, Raj Adhikari (2010) desarrolló un trabajo en el cual el propósito consistió en presentar un concepto de conocimiento para los líderes de la IES para concientizarlos sobre la importancia de la GC para lograr criterios de calidad educativa. 
En su estudio desarrollado para determinar el estado del arte de la gestión del conocimiento en IES Quarchioni, Paternostro \& Trovarelli, (2020) señalan que el interés en investigar la GC en las IES es bastante reciente, en el mismo sentido AlKurdi, El-Haddadeh \& Eldabi (2018) mencionan que es bastante sorprendente que se hayan realizado pocas investigaciones en las instituciones de educación superior, a pesar de que la creación y difusión de conocimientos suelen ser las actividades centrales de estas organizaciones. La GC en las IES se está discutiendo cada vez más (Nawaz, 2015; Quarchioni et al., 2020), esto debido a que éstas deben empezar a repensar su accionar como entes claves en la transformación de la sociedad. Actividades como la producción y transmisión de conocimiento se consideran objetivos tradicionales de las IES (Rowley, 2000) o como lo menciona Zhou \& Tang (2020) históricamente éstas entidades han tenidos dos misiones principales: la investigación y la docencia, cuyos resultados han contribuido a contribuir al desarrollo económico y social. Estas actividades han venido siendo influenciadas por cambios en el entorno tecnológico (Fernández et al, 2018; Quarchioni et al., 2020) y la rápida evolución del mercado global, los cuales son desafíos a los que estas organizaciones se enfrentan a la hora de decidir cómo desarrollar, gestionar y transferir conocimiento (Quarchioni et al., 2020).

Quarchioni et al., (2020) identificaron seis áreas de énfasis sobre las cuales se ha centrado la investigación de la GC en IES, estas son: gestión e informes de capital intelectual para la creación de valor, la transferencia de conocimiento de alto impacto, la adopción de tecnología para apoyar la GC, mejora de los modelos educativos a partir de la GC, producción e intercambio de conocimiento y la adopción de conocimiento. Además, estos autores también proponen continuar con la investigación sobre la interacción entre la dinámica interna de GC y los procesos de transformación relacionados con el entorno institucional, así como también sugieren desarrollar estudios encaminados a mostrar interrelaciones entre las actividades misionales de la IES y como éstas puede ayudar a crear valor para la sociedad.

Para que desde las IES se logre impactar en las problemáticas de la sociedad se hace necesario que las personas apropien el conocimiento que les permita construir 
propuestas de mejoramiento. En este sentido, Cheng (2020) y Almujally \& Joy (2020) desarrollaron estudios sobre la transferencia de conocimiento. La transferencia es un proceso de conocimiento fundamental para efectos de transcender en el cumplimiento misional de las IES, tal como lo señala Cheng (2020) quien afirma que se ha convertido en una de las misiones centrales de las IES, de las cuales se espera que creen activos de conocimiento mediante la realización de actividades de investigación y desarrollo y la transferencia de los conocimientos adquiridos a sus destinatarios.

No obstante, la transferencia es una acción asociada a un destinatario sin mayor variabilidad en un proceso de intercambio de conocimiento. Dicha acción se traduce en el traspaso de habilidades, conocimientos, tecnología, métodos de fabricación o servicios (Vázquez, 2017) que, si bien fortalecen el propósito de la IES sobre la proyección social, se puede quedar en proceso sin mayor posibilidad de transformación. Sin embargo, el conocimiento dinamiza y hace posible que la transferencia pase de lo tácito a lo explícito y de la IES a la sociedad y comunidad, a fin de lograr la transformación de realidades, de modo que, la transferencia es aprehensible siempre y cuando haya comunicabilidad, discusión y creación.

En tal sentido, las IES deben migrar hacia un enfoque donde no solo lo importante sea la generación de productos teóricos de investigación que se hacen necesario, sino que realice una investigación aplicada a las problemáticas territoriales, lo cual seguramente implicará procesos de conocimiento tales como la transferencia asumida como un proceso de intercambio, la sistematización, la protección y el uso del conocimiento, para que a partir de aquí se pueda innovar. En este sentido un sistema de GC sería el marco de referencia sobre el cual se articulen los anteriores procesos. Un sistema de GC puede considerarse como la forma de unir el conocimiento individual, especialmente el conocimiento estratégico, en una organización que aprende (Fernández et al, 2018), enfoque que unido a la necesidad que las IES transfieran conocimiento a la sociedad, daría como resultado un sistema que le permitirá a éstas entidades trascender los roles tradicionales de docencia e investigación, hacia un nuevo enfoque donde la prioridad sea la transferencia como un proceso de intercambio y apropiación social del conocimiento 
para la innovación, dado que como propone Hidalgo \& Albors, (2008) el conocimiento juega un papel crucial en el fomento de la innovación.

En este contexto las IES juegan un papel fundamental para la facilitación o la realización de actividades de participación comunitaria para promover el emprendimiento, innovación y compromiso social, los cuales son los tres ejes vertebrados de lo que se conoce como la tercera misión, es decir que, para lograr la transferencia como un proceso de intercambio y apropiación del conocimiento, es necesario que los actores objeto de las intervenciones apropien herramientas investigativas a fin de establecer acciones que den respuesta a sus realidades y expectativas. De modo que, Meirawan, Prihatin, Suhardan, Dintha \& Komariah (2020) concluyen que la creación de conocimiento de los líderes está íntimamente relacionada con la gestión del conocimiento y las aplicaciones del conocimiento en el diseño y ejecución de planes estratégicos para resolver diversos problemas.

Estos autores también recomiendan que si la educación superior quiere sobrevivir y ser competitiva, depende mucho de la creación de conocimiento, aspecto que es compartido por Raj Adhikari, ( 2010) quien sostiene que el necesario gestionar los aspectos sociales y técnicos de la GC para restaurar y utilizar el conocimiento y elevar el nivel de excelencia en el desempeño de las IES. También, Hidalgo \& Albors (2008) concluyen en su trabajo que es necesario establecer un esquema general junto con los gobiernos nacionales y regionales para promover la gestión de la innovación, la cual debe partir de: la promoción de herramientas, metodologías y el desarrollo de redes para fomentar el intercambio de conocimientos y experiencias; así también, se hace necesario que desde las entidades del gobierno se desarrollen temas relacionados con la sensibilización y actividades para mejorar la confianza de los ciudadanos para fomentar la competitividad.

No obstante, para las IES no es el mercado en si el que las ha puesto en un constante nivel de exigencia, son las condiciones y demandas de un contexto, toda vez que la gestión del conocimiento es quien permite direccionar las actividades de investigación en cuanto a la capacidad de gestión, transferencia como un proceso de intercambio y apropiación de información y resultados de investigación gestados en un área del saber. A su vez, que al estimarse el alcance que tienen estas para 
hacer parte de agendas específicas en la materia, se requiere específicamente, el reconocer el esquema o modelo de gestión de conocimiento que refrenda la entidad o formación en aras de favorecer la transferencia de conocimiento, y de paso, el poder articular dicho conocimiento a los diferentes sectores que efectivamente lo requieren o lo demandan.

"No obstante, cabe destacar que los principios descritos tienen una aplicación más generalizada a cualquier organización, ya sea económica o social, privada o pública, manufacturera o de servicios, en la era venidera, independientemente de su campo de actividades, así como de su ubicación geográfica y cultural. La teoría explica cómo el conocimiento que poseen los individuos, las organizaciones y las sociedades se puede ampliar y enriquecer simultáneamente mediante la amplificación en espiral e interactiva del conocimiento tácito y explícito que poseen los individuos, las organizaciones y las sociedades. La clave para esta expansión sinérgica del conocimiento es la creación conjunta de conocimiento por parte de individuos y organizaciones. En este sentido, la teoría de la creación de conocimiento organizacional es al mismo tiempo una teoría básica para construir una sociedad del conocimiento verdaderamente "humanista" más allá de las limitaciones de la mera "racionalidad económica". Traducido de Nonaka (1994, p. 34).

La gestión del conocimiento permite contribuir para que cada área de las IES termine encadenada (complementada) y eslabonada (valor agregado) conforme al establecimiento de fórmulas y mecanismos de gestión de esta. Esta gestión implica las interacciones entre la IES y el entorno, su capacidad de organización para reaccionar y actuar ante los desafíos y, desde luego, considerar y valorar el impacto de la producción del saber en el entorno, a través de un proceso de retroalimentación estratégica (Greiner et al, 2007). Por ello, el reconocimiento interinstitucional entre las IES y el resto de los estamentos está sujeto a la calidad y cualidad de los procesos académicos y generación de conocimiento sembrados en ellas. Al administrar, gerenciar y promover los logros y procesos derivados de dichas actividades será alcanzable la gestión de conocimiento. 
La estrategia nacional de apropiación social de la ciencia, la tecnología y la innovación (2009) busca ampliar la comprensión de las dinámicas de usos y producción del conocimiento, a partir de generar sinergias ente actores académicos, estatales, comunitarios y productivos. Además de formatear la participación ciudadana en políticas públicas en CTI, promover iniciativas científico-tecnológicas de estos actores que contribuyan al desarrollo humano de las comunidades involucradas a su vez, mecanismos de formación y proyectos y procesos de comunicación sobre ciencia, tecnología, innovación y Sociedad. Por apropiación de conocimiento se comprende un proceso de relaciones entre ciencia y tecnología a partir de participación de actores que generan conocimiento y, por tanto, está caracterizado por una organización e intención, una red de expertos que está en un diálogo. También hay un empoderamiento del conocimiento por parte de la sociedad civil y la interacción es el fundamento de la innovación y, desde luego, de la transformación social.

La Política Nacional de Apropiación Social del Conocimiento de (2020) define cinco principios de la apropiación social del conocimiento: reconocimiento de un contexto, participación, dialogo de saberes y conocimiento, confianza y reflexión crítica. Su propósito es mejorar la calidad de vida de los colombianos a partir de gestionar la articulación entre políticas, planes, programas y proyectos de CTel con los actores del orden nacional, sectorial y territorial. Entre sus lineamientos estratégicas está la generación de procesos, acciones, espacios, capacidades e investigación en apropiación social del conocimiento. De otro lado, el documento borrador CONPES de Política de CTel (2021-2030) propone la ruta estratégica y prospectiva en materia de ciencia, tecnología e innovación para el país, a fin de fortalecer la política de apropiación del conocimiento en:

La política establece seis ejes estratégicos: (i) Fomentar las vocaciones, la formación y el empleo cualificado en la sociedad colombiana; (ii) Desarrollar un entorno habilitante para la generación de conocimiento; (iii) Aumentar el uso del conocimiento en el país; (iv) Incrementar la valoración y apropiación social del conocimiento; (v) Mejorar la gobernanza multinivel del SNCTI e (vi) Incrementar el volumen, la eficiencia y la evaluación de la financiación 
(Consejo Nacional de Política Económica y Social. Documento borrador CONPES, 2020, p. 3)

En cuanto a las Instituciones de Educación Superior IES, su estructura investigativa giró, en gran medida, en torno a la realización de proyectos y trabajos que para la actualidad son considerados como aplicación y/o extensión. De hecho, los lineamientos y políticas de funcionamiento de las IES lo orientan el Ministerio de Educación Nacional, para dotar al sector educativo de un servicio de calidad con acceso equitativo y con permanencia en el sistema. Asimismo, el Ministerio tiene como objetivo, garantizar y promover, a través de políticas públicas, el derecho y el acceso a un sistema educativo público sostenible que asegure la calidad y la pertinencia en condiciones de inclusión, así como la permanencia en el mismo, tanto en la atención integral de calidad para la primera infancia como en todos los niveles: preescolar, básica, media y superior (Lahec, 2012).

Desde luego no es posible hablar de calidad sin extensión, la cual debe buscar conexiones que contribuyan a fortalecer los vínculos de la triada, empresauniversidad-estado, combinación necesaria para alcanzar el desarrollo. La extensión se constituye así en un canal de comunicación que permite: conocer las innovaciones producidas en los países más avanzados, establecer redes para realizar las mejores prácticas, transferir conocimientos, retroalimentar el proceso docente educativo, dinamizar la movilidad académica, fortalecer las prácticas empresariales, acercar los graduados con la academia, constituyéndose en carta de presentación ante el ámbito donde operan sus procesos educativos. Los anteriores son retos que deberá enfrentar la universidad contemporánea (Botero, 2009 , p. 6).

\section{Discusión}

El papel que debe cumplir la investigación en las IES, además de las concebidas en materia de producción de artículos y ponencias, no se centra en el desarrollo académico, la extensión e internacionalización de las IES, sino la producción, gestión y apropiación del conocimiento mediante la implementación del sistema de gestión de conocimiento que posibilite fortalecer y generar capacidades 
institucionales y permita el fortalecimiento del capital humano, la transferencia como un proceso de intercambio y apropiación de conocimiento. En este sentido, las IES, en cuanto a investigación, innovación, ciencia, tecnología, entre sus propósitos considera crear, desarrollar y difundir el conocimiento científico y tecnológico, orientado a consolidar los campos del saber propios de las disciplinas que reflexionan.

De hecho, la ruta de investigación para las IES considera la Misión de Sabios (2019) la cual fue convocada por el presidente de Colombia para temas de ciencia, tecnología, innovación, educación y desarrollo, que entre sus propósitos está la apropiación social del conocimiento a través del fortalecimiento de un sistema de innovación transformativa para el desarrollo local, desde el conocimiento y en clave de los Objetivos del Desarrollo Sostenible.

Las estrategias de apropiación social del conocimiento estarán enfocadas a difundir los nuevos desarrollos científicos y tecnológicos, de manera que no solo se promueva la adopción de innovaciones recientes, sino que se logre la participación activa de diferentes grupos sociales en procesos de innovación (Misión de Sabios, 2020, p.106)

Esta Misión hace énfasis en la urgencia de realizar acciones e iniciativas que permitan afrontar el entorno económico global que es complejo e incierto, a partir la oportunidad que tiene Colombia de convertirse en líder tecnológico internacional en algunos "nichos de biotecnología, industrias creativas e industria 4.0, aprovechando sus dotaciones de suelo, agua y recursos hidrobiológicos, biodiversidad, radiación solar y localización, su diversidad cultural, las capacidades de sus principales universidades y egresados, los conocimientos ancestrales y los vínculos con la diáspora de talentos vinculados a entidades investigativas de primer nivel" (Misión de Sabios, 2019, p.8)

La principal misión de la gestión del conocimiento es crear un ambiente en el que el conocimiento y la información disponibles en una organización sean accesibles y puedan ser usados para estimular la innovación y hacer posible mejorar las decisiones. La clave estaría en crear una cultura. La gestión del conocimiento rinde sus frutos cuando las organizaciones no se apoyan únicamente en soluciones 
tecnológicas; por el contrario, se debe reconocer la importancia del factor social y de las conexiones humanas que se necesitan para compartir el conocimiento (Blas \& Tamanini, 2009, p. 26).

La docencia, la extensión y la misma internacionalización no registran los proyectos o trabajos de investigación como centro de su saber. En el país, la transferencia de conocimiento por cuenta de los resultados investigativos no alcanza ni a contemplarse en los escaños de los doctorados y las maestrías. Ha sido difícil establecer el medio por el cual establecer los procesos de irrigación sobre los cuales está se extendería ampliamente. La investigación ha alcanzado a incubarse en la realidad institucional de las IES y, desde luego, la base para la apropiación del conocimiento es la investigación, a partir de la organización intencionada de métodos y saberes, cuyo fin es la conformación de una red sociotécnica de expertos en ciencia y tecnología y grupos sociales que posibilite un conocimiento del cual se empodere la sociedad civil. Para ello:

La investigación tiene como principales objetivos, la generación de conocimiento, a través de la producción de nuevas ideas; y la solución de problemas prácticos. Sin embargo, hay que pensar en ella como un proceso, en el que han de tomarse en consideración, y de forma rigurosa, diferentes etapas sin prescindir de ninguna de ellas. (Manterola \& Otzen, 2013, p.1498) De este modo, la gestión del conocimiento (Ver figura 1) y los procesos comunicacionales son el epicentro de las actividades innovadoras, debido a que estas, las podemos entender como la capacidad de una empresa para generar nuevos conocimientos, difundirlo entre sus empleados y materializarlos en productos 0 en mejoras organizacionales. Estas innovaciones (radicales 0 incrementales) no se dan por espontaneidad, sino por la gestión de la alta gerencia, con el acompañamiento de sus colaboradores, a través de políticas y operaciones que dan respuestas eficaces a problemáticas reales y/o potenciales en los procesos (Fontalvo et. al, 2011) 
Fig. 1. Tipologías de modelos para la GC (Rodríguez, 2006).

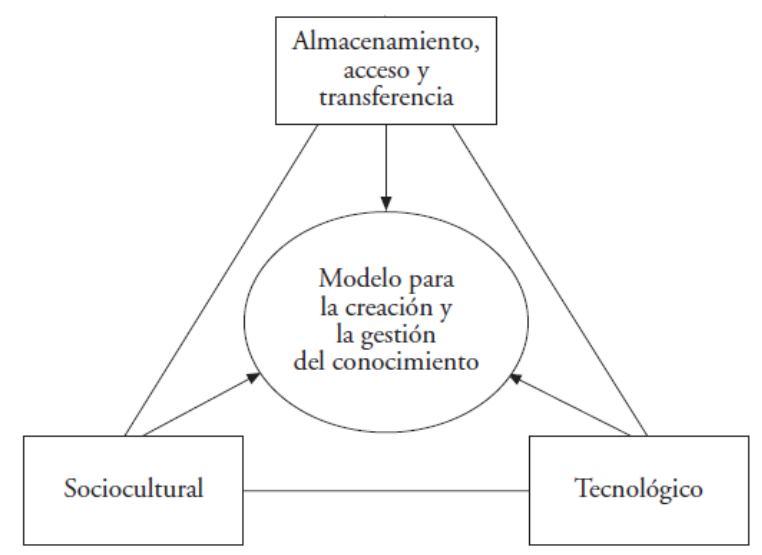

La transferencia de la investigación en sentido estricto inicia cuando el profesor adscrito en docencia acoge los artículos, proyectos y seminarios abarcados por el docente investigador. La oportunidad de convertir el conocimiento adquirido en criterio formulador de los cuestionamientos o preguntas que deben recrear los aspectos relacionados en las aulas representa la exclusiva y principal contribución a saber. El profesor universitario no está obligado a repensar su quehacer en tanto los fines de la docencia, pero si debe observar los parámetros que contempla la investigación como una forma de desarrollarla. La gestión de proyectos de investigación desarrollados en las IES debe superar el escaño de la ejecución interna. No obstante, la sinergia que pueden crear los propios centros, direcciones o vicerrectorías de investigación hacia afuera puede ser más productivo en tanto gestión y resultados, que el esperar que las dinámicas externas indiquen cuando actuar. Aún en la investigación de las IES, la pasividad positiva, es un reflejo de lo que se tienen a la hora de evaluarla.

Las IES como concepto de organización, hoy por hoy, no puede exponerse sin el valor del capital intelectual, las estrategias propuestas en cualquier modelo de planeación no pueden discriminar la gestión del conocimiento como fuente única de diferenciación que posibilita la competitividad y sostenibilidad de las empresas, de este modo, la revisión y apropiación de los referentes teóricos de la gestión de conocimiento sobre los cuales se sustente una estrategia empresarial orientada a 
la creación de una cultura organizacional de conocimiento, permite establecer criterios que concretan la creación de valor a través de la gestión de conocimiento. Para lograrlo se requiere esbozar las capacidades para convertir una organización que crea valor a partir de la gestión de conocimiento (Tarazona \& Mediana, 2009) La búsqueda de autonomía para la investigación en las IES es alcanzable en la medida en que se reconvierta el canon de estructura endógena que raya con la endogamia. Si en principio es acertada la discrecionalidad en cuanto a lo referente a autorías, desarrollos y patentes, la realidad indica que al respecto se ha diseñado el segundo piso sin comprender ampliamente el primero. La generación, impacto y transferencia del conocimiento debe ser evaluada como fórmula para la obtención de apoyos y recursos económicos, no solo, en la consumación de un descubrimiento, hallazgo o producto, en donde existen dificultades para lograr el cometido necesario antes que el mercado lo indique.

Es preciso establecer vínculos/alianzas con centros de investigación y otras organizaciones, con la finalidad de diversificar/fortalecer las líneas de investigación en la perspectiva de trabajos multi y transdisciplinarios, todo ello, para impulsar la producción, promoción, difusión y transferencia de conocimientos, para lo cual es necesario, más no suficiente, disponer y utilizar los recursos tecnológicos adecuados a los objetivos y planes en materia de GC e investigación. (Yánez \& Yánez, 2013, p. 118).

En esa medida, la gestión del conocimiento en las IES en Colombia para la apropiación social del conocimiento se inscribe en la identificación de situaciones y fenómenos, también en la identificación de capacidades y potencialidades de los actores sociales, productivos y gubernamentales que requieren una transformación de sus procesos, a partir de las nuevas prácticas, relaciones, saberes y conocimientos. Para ello se requiere la generación de nuevas estrategias e instrumentos en red que son posible desde la investigación, a fin de consolidar una sociedad del conocimiento, en el que cada actor es capaz de comunicar saberes y generar conocimiento, en especial, que estos sean apropiados socialmente:

En cuanto a la antigua dimensión vertical, la contribución ciudadana invierte el flujo unidireccional del modelo anterior, convirtiéndolo en un flujo 
multidireccional y multicéntrico, haciendo incluso que deje de ser aplicable la separación dimensional presente. Este nuevo modelo, más diversificado y rico en conexiones, estaría representado más satisfactoriamente por una topología en red. Esta estructura apoya la posibilidad de plantear las cuestiones de la generación de conocimiento en ciencia y tecnología en términos de redistribución, como proponíamos al principio de este trabajo (Barrios, 2008)

De modo que, la gestión del conocimiento en las IES para la apropiación social del conocimiento implica que estas se reconozcan como instituciones capaces de generarlo en primera instancia, pero para el efecto, se requiere de los procesos de autoevaluación y certificación provenientes de las mismas mediciones del mercado, a su vez, establecer indicadores y valoraciones de impacto del conocimiento generado en la sociedad. Dicha etapa amplía la capacidad de observancia que pueden tener las IES para superar sus dificultades básicas y ubicarse en la línea de mejores prácticas. Es decir, el salto de la docencia a la investigación y, por ende, de la extensión e impacto con la relación Universidad, Empresa y Estado, incrementan tanto a la generación como a la gestión de conocimiento como preámbulo, que además de ser incorporada, pasará a ser prueba de su propia función en cuanto al impacto que en adelante debe generar en la actividad económica y productiva en donde tiene asiento.

En tal sentido, una ruta metodológica posible en y para la gestión del conocimiento en las IES para la apropiación social del conocimiento es el análisis Multicriterio; permite comparar alternativas frente a una temática que pueden ser cualitativas 0 cuantitativas (Belacel, 2000) y establecer la argumentación en áreas prácticas, sociales y teoréticas en las cuales debe sostenerse la idea del por qué establecer además de buenas prácticas en materia de gestión del conocimiento, el sistema que las soporte. El análisis Multicriterio permite el abordaje de la gestión del conocimiento mediante la producción y aplicación de la ciencia, tecnología e innovación desde dos perspectivas. Primera, toma en cuenta cifras, datos, evaluaciones, gráficos, mapas, modelos, pruebas estadísticas y de ajuste como parte de las referencias que deben tenerse a la hora de abordar un tema en áreas 
de conocimiento de las IES, en clave de identificación, interacción y relación entre actores: gobierno, académica, sector productivo y comunidades.

Segunda perspectiva, convoca a estos actores a dialogar e intercambiar, conocimiento, experiencias y saberes a partir de la generación de entorno de confianza e inclusión de realidades que se requieren transformar para generar un bienestar social. Seguido de ello, se apoya en el abordaje académico, evidencia empírica, teoría y teóricos para afianzar paso a paso las razones del problema o tema a considerar en el marco del proyecto o propuesta de estudio a saber.

La potencialidad del análisis multicriterio es cualitativo y cuantitativo, por cuanto en esencia, toma a ambos como frentes de trabajo para poder alistar los argumentos que requiere para postular en cada uno de ellos, los criterios sobre los cuales se pretende apoyar el problema o tema de estudio en cuestión: la transferencia y apropiación social del conocimiento. En este sentido, el análisis multicriterio se convierte en un frente de trabajo, más que una propia metodología, por cuanto en su haber está dirigido a ofrecer elementos de trabajo sobre los cuales pueda enriquecerse el tema de investigación y la apropiación del conocimiento mediante el diálogo y relación entre la academia, el gobierno, el sector productivo y las comunidades; en especial, este último actor:

(...) aporta ideas desde diferentes áreas del conocimiento aprendido formalmente (física, química, informática, biología, ...), o de manera no formal y experiencial (siembra de cultivos, manejo de materiales naturales para construcción, disposición adecuada de residuos sólidos, uso de plantas medicinales, ...) y reconocer, valorar, comprender, usar y aplicar ese conocimiento para, de manera colectiva, atender nuestras necesidades con recursividad, ingenio y sentido de pertenencia (Minciencias, 2020, p.7)

En este sentido, el análisis multicriterio para la gestión del conocimiento considera cuatro variables: primera, la dirección de las actividades de investigación de las IES hacia la capacidad de transferencia y aprobación de información y resultados de investigación gestados en un área del saber, en especial, hacia la puesta en marcha de proyectos y áreas de interés social y económica que requiere de una apropiación social del conocimiento (Figura 2). Segunda, el papel que tienen las IES como parte 
de agendas específicas del CTel, se requiere que reconozcan su esquema o modelo de gestión de conocimiento en aras de favorecer la transferencia de conocimiento, y de paso, el poder articular el mismo a los diferentes sectores que efectivamente lo requieren o lo demandan. Este es el caso de la administración pública, formación que en materia de gestión y formulación de la agenda en CTel está en ciernes.

Fig. 2. Apropiación Social del Conocimiento.

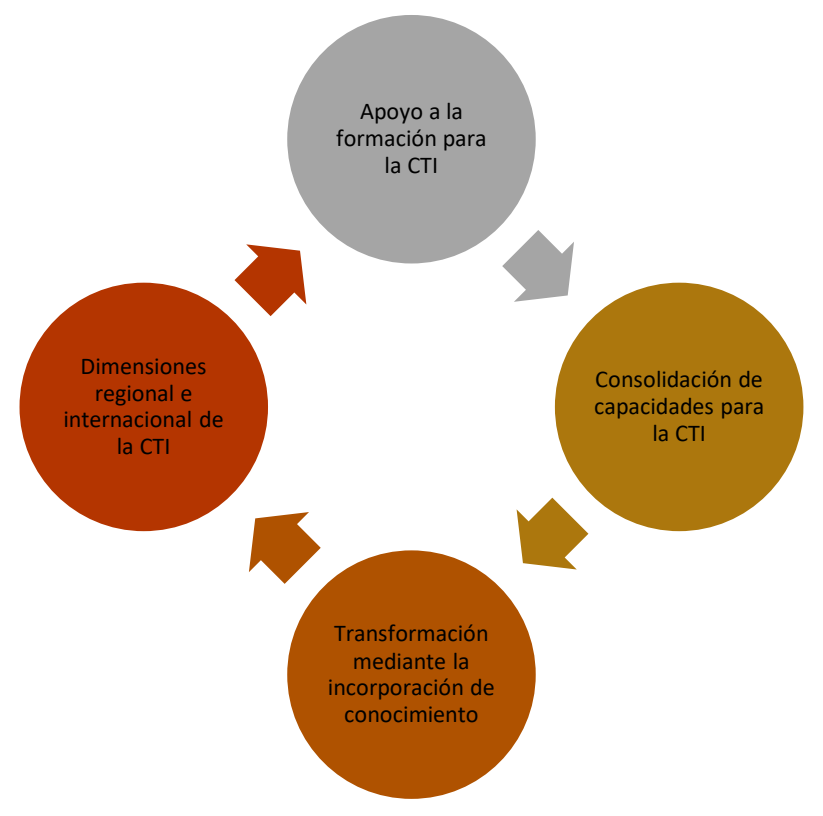

Tercera variable, el reconocimiento interinstitucional entre las IES y el resto de los estamentos está sujeto a la calidad y cualidad de los procesos académicos y generación de conocimiento sembrados en ellas. Al administrar, gerenciar y promover los logros y procesos derivados de dichas actividades será alcanzable la gestión de conocimiento. A trazo seguido, la cuarta variable es la retroalimentación externa e interna que pueda lograrse bajo dicho precepto hará posible que las IES, pasen de depender de la cobertura en educación por la de conocimiento, esto es, estructurar el sistema de gestión de conocimiento como columna transversal del quehacer institucional en todas las áreas sustantivas y de su razón misional. El sistema permitiría además de elevar la gestión y los procesos autoevaluados y evaluados con fines de acreditación y calidad, a incrementarla como punto de referencia interinstitucional para el desarrollo de actividades afines a ella. Etapa, 
que depende de la integralidad y permeabilidad de dicho sistema de gestión a todas las áreas y divisiones institucionales.

El sistema de gestión del conocimiento pondría a cada uno de los estamentos de manera directa e indirecta a ponderar a las unidades administrativas, directivas y de gestión con cargo a la gestión del conocimiento, repensándolas igualmente como unidades que conforme a dicho criterio podrían igualmente convertir su conocimiento y experiencia en centros desde los cuales podría transferirse igualmente conocimiento. No solo se compartiría conocimiento en sentido estricto, sino que el contenido que privilegia la gestión del conocimiento atiende precisamente el impacto y la trascendencia que tiene la investigación o los resultados análogos de la misma en aras de contribuir al mejoramiento y/o solvencia de los problemas, o a la capacidad crítica y propositiva, que tienen las áreas del saber para incubar el conocimiento formal en las aplicaciones metodológicas y prácticas de quienes se sirven de ello para cumplir sus metas o propósitos.

\section{Conclusiones}

Es sumamente necesario que las calidades y condiciones adquiridas en cuanto a conocimiento tengan asiento en el marco de alianzas, encuentros y propuestas emergidas con los diversos actores que expresan también las bondades de elevar los esfuerzos en cuanto a la generación de conocimiento hacia los sistemas de gestión en los que la articulación entre ellos estaría cimentada en la definición de fines e intereses concretos. La gestión del conocimiento implica para las IES el reconocerse como entidades que generan, impactan, transfieren y promueven el conocimiento como un proceso de intercambio, y no se limitan a su adquisición como sucede en la actualidad. Por tanto, el disponer de una unidad de gestión de proyectos estratégicos requiere que, a nivel ejecutivo, la gestión del conocimiento genere resultados tanto en docencia como investigación, pero, en especial, en generar y establecer diálogos con el gobierno y las comunidades, a fin de fortalecer capacidades en la sociedad para la toma de decisiones, la participación ciudadana, promover iniciativas de transferencia como un proceso de intercambio de 
conocimiento científico para el desarrollo integral de las necesidades y expectativas de las comunidades y de los territorios involucrados.

El primer piso de la educación superior universitaria ha sido superado, tal es así que la docencia como referente hoy es abierta y ofertada desde diferentes formas pedagógicas para la formación de un saber. No obstante, el segundo piso, relacionado con la investigación y generación de conocimiento aún se encuentra en ciernes. De allí entonces, que para poder establecer las bases sólidas de la gestión del conocimiento dichos pasos deben cumplirse, para luego, transformar administrativamente las IES para poder transformase en instituciones de gestión del conocimiento

Es crucial replantear el camino que sigue en la generación y transferencia del conocimiento al interior de las IES, el cual está a cargo de los grupos de investigación, pero, debe entenderse que estos necesitan alcanzar un cierto margen de autonomía administrativa que contemplada, observada o verificada puede traer frutos interesantes. De igual manera, la generación y transferencia como un proceso de intercambio del conocimiento desde las IES es hacia los sectores productivos, la sociedad civil y el gobierno mediante la gestión de los restos que implica la producción del conocimiento a partir de la investigación cuya estructura requiere la formación y vinculación de capital humano en Investigación, Desarrollo e Innovación I+D+l, además de principios y enfoques como: Transversalidad, Anticipación o prospectiva, Direccionalidad enfocando recursos y esfuerzos 4. Transformación a partir de establecer cambios en los sistemas sociotécnicos y fomentar el desarrollo de nuevos mercados basados en innovación. 5. Territorialidad. 6. Sostenibilidad (Documento borrador CONPES, 2020).

\section{Referencias}

Al-Kurdi, O., El-Haddadeh, R., Eldabi, T. (2018). Knowledge sharing in higher education institutions: a systematic review. Journal of Enterprise Information Management, 31(2), 226-246. https://doi.org/10.1108/JEIM-09$\underline{2017-0129}$

Almujally, N., Joy, M. (2020). A Knowledge Sharing System Architecture for Higher Education Institutions. In: Kumar V., Troussas C. (eds) Intelligent Tutoring Systems. ITS 2020. Lecture Notes in Computer Science, 12149, 397-40. https://doi-org.ezproxy.eafit.edu.co/10.1007/978-3-030-49663-0 48

Banco Mundial. (1998). Informe sobre el desarrollo mundial 1998/1999: Conocimiento para el desarrollo. Nueva Pensamiento y Acción, Tunja (Boyacá-Colombia) - No. 31. Julio - Diciembre 2021. 
Gestión del conocimiento en las Instituciones de Educación Superior para la apropiación social del conocimiento

York: Oxford University Press. https://openknowledge.worldbank.org/handle/10986/5981

Barrios, C. (2008). La apropiación social de la ciencia: nuevas formas. Revista CTS, (4)10, 213-225. https://www.redalyc.org/pdf/924/92441014.pdf

Belacel, N. (2000). Multicriteria assignment method PROAFTN: Methodology and medical application. European Journal of Operational Research, 125(1), 175-183

Blas, L., Tamanini, H. F. (2009). Gestión del conocimiento, ¿de qué estamos hablando?. Petrotecnia, 12-26. http://www.petrotecnia.com.ar/junio09/gest.conoc Blas.pdf

Botero, C. (2009). Cinco tendencias de la gestión educativa. Revista Iberoamericana de Educación. EDITA: Organización de Estados Iberoamericanos para la Educación, la Ciencia y la Cultura (OEI). 49(2), 1-11. https://doi.org/10.35362/rie4922100

Cheng, E. C. K. (2020). Knowledge transfer strategies and practices for higher education institutions. VINE Journal of Information and Knowledge Management Systems, 11, e0184. https://doi.org/10.1108/VJIKMS11-2019-0184

Chong, S. C., Salleh, K., Ahmad, S. N. S., Sharifuddin, S. I. S. O. (2011). KM implementation in a public sector accounting organization: An empirical investigation. Journal of Knowledge Management, 15(3), 497-512. https://doi.org/10.1108/13673271111137457

Colciencias (2009). Estrategia nacional de apropiación social de la ciencia, la tecnología y la innovación. Disponible: https://minciencias.gov.co/sites/default/files/upload/paginas/estrategianacional-ascti.pdf

Consejo Nacional de Política Económica y Social. Documento borrador CONPES de Política de CTel (20212030). Política Nacional de Ciencia, Tecnología e Innovación 2021-2030. https://minciencias.gov.co/sites/default/files/documento conpes ciencia tecnologia e innovacion.pdf

Escorcia, J., Barros, D. (2020). Gestión del conocimiento en Instituciones de Educación Superior: Caracterización desde una reflexión teórica. Revista de Ciencias Sociales, XXVI(3), 83-97. https://doi.org/10.31876/rcs.v26i3.33235

Fernández-López, S., Rodeiro-Pazos, D., Calvo, N., Rodríguez-Gulías, M. J. (2018). The effect of strategic knowledge management on the universities' performance: an empirical approach. Journal of Knowledge Management, 22(3), 567-586. https://doi.org/10.1108/JKM-08-2017-0376

Fontalvo, H. T. J., Quejada, R., Puello, G. (2011). La gestión del conocimiento y los procesos de mejoramiento. Dimensión Empresarial, 9 (1), 80-87. https://dialnet.unirioja.es/servlet/articulo?codigo=3797779

Greiner, M., Böhmann, T., Krcmar, H. (2007). A strategy for knowledge management. Journal of Knowledge Management, 11 (6), 3-15. https://doi.org/10.1108/13673270710832127

Hidalgo, A., Albors, J. (2008). Innovation management techniques and tools: A review from theory and practice. $R \& D$ Management, 38(2), 113-127. https://doi.org/10.1111/j.1467-9310.2008.00503.x

Landoli, L., Zollo, G. (2006). Organizational cognition and learning: Building systems for the learning organization. In: Organizational Cognition and Learning: Building Systems for the Learning Organization. https://doi.org/10.4018/978-1-59904-313-5

Laal, M. (2011). Knowledge management in higher education Marjan. Procedia Computer Science, 3, 544-549. https://doi.org/10.1016/.jprocs.2010.12.090

Lin, H., Lee, G. (2006). Effects of socio-technical factors on organizational intention to encourage knowledge sharing. Management Decision, 44(1), 74-88. https://doi.org/10.1108/00251740610641472

Manterola, C. Otzen, T. (2013). Por qué Investigar y Cómo Conducir una Investigación. International Journal of

Pensamiento y Acción, Tunja (Boyacá-Colombia) - No. 31. Julio - Diciembre 2021. ISSN 0120-1190 -eISSN 2619-3353

DOI: https://doi.org/10.19053/01201190.n31.2021.12492 
Gleidy-Alexandra Urrego-Estrada; Jahir-Alexander Gutiérrez-Ossa; Diego-Armando Jurado-Zambrano

Morphology, 31 (4), 1498-1504. https://doi.org/10.4067/S0717-95022013000400056

Meirawan, D., Prihatin, E., Suhardan, D., Dintha, R., Komariah, A. (2020). Creación de conocimiento de líderes de educación superior para enfrentar los cambios. International Journal of Advanced Science and Technology, 29(5s), 1051-1064

Minciencias (2019). Colciencias. https://minciencias.gov.co/sites/default/files/plegableweb.pdf

Minciencias (2020). Política Nacional de Apropiación Social del Conocimiento. https://minciencias.gov.co/sites/default/files/documento de lineamientos para la politica nacional de apropiacion social del conocimiento 1.pdf

Misión de Sabio (2019). Propuestas de la Misión Internacional de Sabios 2019. https://www.minciencias.gov.co/sites/default/files/upload/paginas/propuesta-sabios-txt y portadaalta.pdf

Nawaz, N. (2015). Review of Knowledge Management in Higher Education. International Journal of Science and Research (IJSR), 4(11), 2062-2065. https://doi.org/10.21275/v4i11.nov151609

Nonaka, I. (2007). The Knowledge-Creating Company. Harvard Business Review,165, 162-171.

Nonaka, I. (1994). A Dynamic Theory of Organizational Knowledge Creation. Organization Science, 5 (1), 1437.

Quarchioni, S., Paternostro, S., Trovarelli, F. (2020). Knowledge management in higher education: a literature review and further research avenues. Knowledge Management Research and Practice, Pre-print. https://doi.org/10.1080/14778238.2020.1730717

Raj Adhikari, D. (2010). Knowledge management in academic institutions. International Journal of Educational Management, 24(2), 94-104. https://doi.org/10.1108/09513541011020918

Rodríguez, D. (2006). Modelos para la creación y gestión del conocimiento: una aproximación teórica. Universidad Autónoma de Barcelona. Educar (37), 25-39

Rowley, J. (2000). Is higher education ready for knowledge management? International Journal of Educational Management, 14(7), 325-333. https://doi.org/10.1108/09513540010378978

Tarazona, G., Silva, J., Medina, V. (2009). Generación de Valor en la Gestión del Conocimiento. In: Seventh LACCEI Latin American and Caribbean Conference for Engineering and Technology.

Vázquez, R. (2017). Transferencia del conocimiento y tecnología en universidades. Revista de Ciencias $\begin{array}{llll}\text { Sociales } \quad y & \text { Humanidades, } & \text { 75-95. }\end{array}$ https://doi.org/10.28928/revistaiztapalapa/832017/atc3/vazquezgonzalezer

Von Krogh, G. (1998). Care in knowledge creation. California Management Review, 3, 133-153. https://doi.org/10.2307/41165947

Yánez, J., Yánez, R. (2013). Gestión de conocimiento: Un modelo para impulsar la investigación en los postgrados. Universidad del Zulia. Revista Venezolana de Gerencia. 18 (61), 105-120. https://www.redalyc.org/pdf/290/29026161005.pdf

Zhou, R., Tang, P. (2020). The role of university Knowledge Transfer Offices: Not just commercialize research outputs! Technovation, 90-91, e102100. https://doi.org/10.1016/j.technovation.2019.102100

Pensamiento y Acción, Tunja (Boyacá-Colombia) - No. 31. Julio - Diciembre 2021. ISSN 0120-1190 -elSSN 2619-3353

DOI: https://doi.org/10.19053/01201190.n31.2021.12492 